\title{
Note on an Apparatus for Determining the Quantity of Dissolved Gases in Sea Water, and in Fluids containing Organic Matter.
}

\author{
By \\ H. W. Harvey, M.A. \\ Hydrographer at the Plymouth Laboratory.
}

With Figures I-IV in the Text.

An apparatus was devised with the primary object of determining the atmospheric nitrogen and oxygen dissolved in sea water from on board ship in fine weather. It was found that the apparatus could be used at sea in fine weather, but owing to the difficulty of manipulation when any sea was running there was a possibility of error which necessitated duplicate determinations being made, each of which took about twenty minutes. In the Laboratory, on the other hand, the apparatus gives rapid and reasonably accurate results, and may be of use for the estimation of oxygen or other dissolved gases in fluids which contain organic matter, rendering Winkler's method of oxygen determination impossible, and where there is an insufficient quantity of fluid and time to carry out extraction of the gases by means of a mercury pump.

The apparatus was made as shown diagramatically in Fig. I, consisting of a glass bulb filled with mercury, with the necessary means of evacuating it and trapping any air leak which may occur in the process. It is mounted in a weighted teak box with hinged door and can be let down by a line to any desired depth in the sea, the tap $t$ opened by a messenger and the charge of sea water ( 4.59 c.c. in this particular apparatus) drawn in, as shown in Fig. II. The apparatus is then drawn up, tap $t$ and screw pinch cock s closed, and the bulb evacuated by lowering the mercury reservoir and opening tap $q$ (Fig. III). Tap $q$ is then closed and the apparatus shaken for two minutes. This is easily done by clamping the hinged door of the case to a vertical support and swinging the case to and fro.

The pressure is then restored by raising the mercury reservoir, and the resultant bubble of gas forced through the fine bore pressure tubing $x$ into the capillary of the gas analyser (Fig. IV), where it collects at the top of the bulb B. The rubber plug $r$ is then closed, disconnecting the bulb 
and capillary from the water in the jacket $J$ surrounding the gas analyser, the apparatus inverted, and by screwing $\mathrm{B}$ on to the rubber nipple $n$, filled with water, the bubble of gas is forced into the graduated capillary. The end of the capillary is dipped into a 40 per cent $\mathrm{NaOH}$ solution, when by screwing and unscrewing $\mathrm{B}$ the $\mathrm{NaOH}$ solution is drawn into

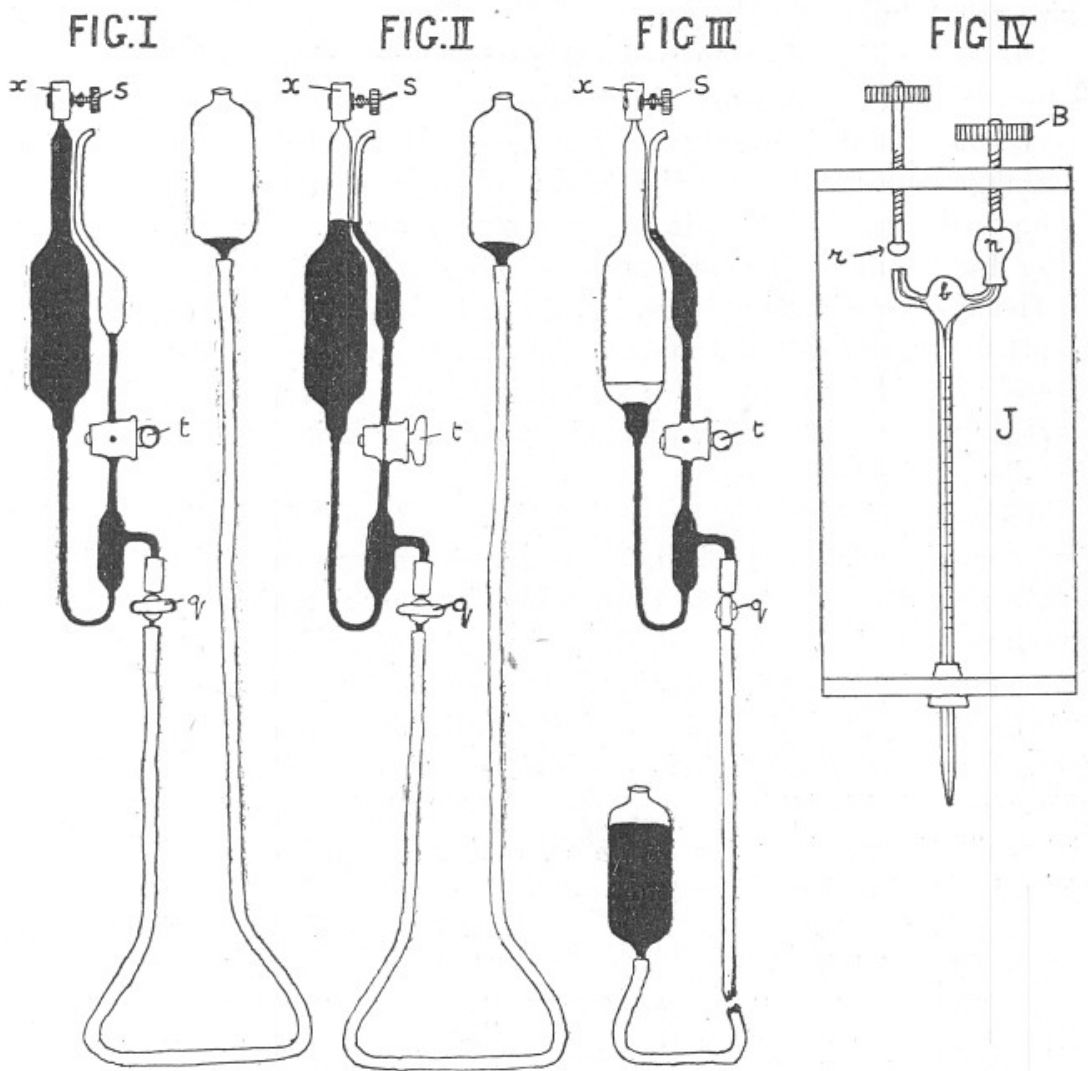

FIG. I.-Extraction apparatus ready to receive charge of fluid.

FIG. II.-With charge of fluid drawn in.

FIG. III.-With Torricellian vacuum above the fluid.

FIG. IV.-Apparatus for analysis of the bubble of gas extracted from the charge of fluid, surrounded by a water-jacket $\mathrm{J}$.

the capillary and the bubble of gas washed in it, the carbon dioxide being absorbed.

In the case of sea water the gas extracted consists of oxygen atmospheric nitrogen and a part only of the carbon dioxide.

The length of the column of residual oxygen and atmospheric nitrogen extracted from the 4.59 c.c. of sea water is then read, together with the temperature of the water jacket of the gas analyser, and its volume at 
N.T.P. calculated. The column of gas is washed in the same manner in alkaline pyrogallol and the residual volume of atmospheric nitrogen noted. To clean out the capillary and bulb of the gas analyser ready for the next estimation, all that is necessary is to open $r$, when fresh water from the jacket $J$ washes out the alkaline pyrogallol remaining, together with the column of nitrogen.

One evacuation of a charge of water extracts practically all the dissolved gases, the partial pressure of the gases in the Torricellian vacuum being very small, and equilibrium being nearly attained after two minutes' shaking. A second extraction gives only a very minute bubble, which, composed mainly of $\mathrm{CO}_{2}$ in the case of sea water, does not materially alter the result of the estimation of oxygen and nitrogen.

The above estimation takes twenty to twenty-five minutes at shallow depths up to twenty fathoms. The charge can also be taken from a vessel of fluid by means of a siphon inserted into the fine bore rubber capillary tube $x$ in Fig. I, when the whole estimation can be completed in fifteen minutes.

With regard to the accuracy of the results obtained, the following two experiments are typical. A vessel of sea water at $15^{\circ} \mathrm{C}$., chlorine content 1.95 per cent, was thoroughly shaken with air at atmospheric pressure (bar. $767 \mathrm{~mm}$.) and allowed to stand for four hours at $15^{\circ} \mathrm{C}$. in a constant temperature bath. The content of oxygen and atmospheric nitrogen in sea water at varying temperatures has been tabulated from a very careful series of experiments by Charles Fox (Publication de Circonstance, No. 41. Copenhagen, 1907), and according to these determinations this water at $15^{\circ} \mathrm{C}$. and $767 \mathrm{~mm}$. barometric pressure should contain when saturated:-

$5 \cdot 86$ c.c. at N.T.P. of oxygen per litre of water.

$11 \cdot 25,, \quad$ nitrogen ,, ,

From three determinations made by the apparatus described it was found to contain :-

$5 \cdot 6$ c.c. at N.T.P. of oxygen per litre of water.

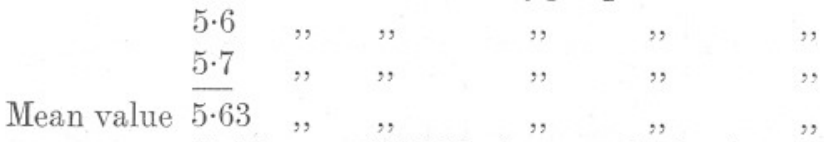

11.55 c.c. at N.T.P. of atmospheric nitrogen per litre of water

$11 \cdot 3$

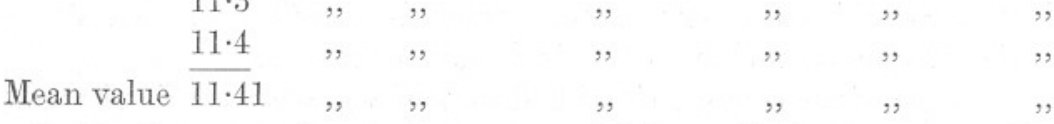

" $", \quad$,

In another experiment water was withdrawn by means of a siphon from a large vessel of sea water at room temperature after thorough 
mixing, and five determinations of its oxygen and nitrogen content were made with the above apparatus and of its oxygen content by means of Winkler's method.

\begin{tabular}{|c|c|c|c|}
\hline \multirow{2}{*}{\multicolumn{2}{|c|}{$\begin{array}{c}\text { Oxygen in c.c. at } \\
\text { N.T.P. per litre of } \\
\text { water. } \\
\text { By above } \\
\text { apparatus. }\end{array}$}} & & $\begin{array}{l}\text { Atmospheric nitrogen in } \\
\text { c.c. at N.T.P. per litre } \\
\text { of water. }\end{array}$ \\
\hline & & $\begin{array}{l}\text { By Winkler's } \\
\text { method. }\end{array}$ & $\begin{array}{l}\text { By above } \\
\text { apparatus. }\end{array}$ \\
\hline & $4 \cdot 62$ & $4 \cdot 86$ & $10 \cdot 31$ \\
\hline & $4 \cdot 74$ & $4 \cdot 85$ & $10 \cdot 31$ \\
\hline & $4 \cdot 62$ & $4 \cdot 85$ & $10 \cdot 54$ \\
\hline & $4: 62$ & $4 \cdot 84$ & $10 \cdot 43$ \\
\hline & $4 \cdot 62$ & $4 \cdot 77$ & $10 \cdot 19$ \\
\hline Mean value & de $4 \cdot 64$ & $4 \cdot 83$ & $10 \cdot 36$ \\
\hline 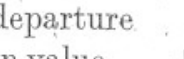 & & & \\
\hline alue & $+0 \cdot 10$ & -0.06 & $+0 \cdot 18$ \\
\hline
\end{tabular}

I am indebted to Mr. C. C. Stockman for very carefully standardising the sodium thiosulphate solution used in the determinations by Winkler's method, and also for making several determinations by the same method as a check upon my own. 